\title{
Analysis of the scattering by an unbounded rough surface
}

\section{Peijun Li ${ }^{* \dagger}$ and Jie Shen}

\section{Communicated by A. Kirsch}

This paper is concerned with the mathematical analysis of the solution for the wave propagation from the scattering by an unbounded penetrable rough surface. Throughout, the wavenumber is assumed to have a nonzero imaginary part that accounts for the energy absorption. The scattering problem is modeled as a boundary value problem governed by the Helmholtz equation with transparent boundary conditions proposed on plane surfaces confining the scattering surface. The existence and uniqueness of the weak solution for the model problem are established by using a variational approach. Furthermore, the scattering problem is investigated for the case when the scattering profile is a sufficiently small and smooth deformation of a plane surface. Under this assumption, the problem is equivalently formulated into a set of two-point boundary value problems in the frequency domain, and the analytical solution, in the form of an infinite series, is deduced by using a boundary perturbation technique combined with the transformed field expansion approach. Copyright $\odot 2012$ John Wiley \& Sons, Ltd.

Keywords: Helmholtz equation; variational formulation; rough surface scattering; boundary perturbation method; transformed field expansion

\section{Introduction}

This paper is concerned with the mathematical analysis of the solution for an acoustic wave scattering problem by an unbounded penetrable rough surface. An unbounded rough surface is a nonlocal perturbation of an infinite plane surface such that the whole surface lies within a finite distance of the original plane. The problem studied in this work falls in the class of rough surface-scattering problems, which arise from various applications in industry and military, such as modeling acoustic and electromagnetic wave propagation over outdoor ground and sea surfaces, optical scattering from the surface of materials in near-field optics or nano-optics, and detection of underwater mines, especially those buried in soft sediments. These problems have received much attention and been intensively examined by researchers in the engineering community. A considerable amount of information is available concerning their solutions via both rigorous methods of computation and approximate, asymptotic, or statistical methods, for example, the reviews and monographs by Ogilvy [1], Voronovich [2], Saillard and Sentenac [3], Warnick and Chew [4], DeSanto [5], Elfouhaily and Guerin [6], and references cited therein.

We study the acoustic wave propagation problem of the Helmholtz equation with an unbounded penetrable scattering surface. Specifically, we consider the scattering of a time-harmonic wave field, generated from a point source, incident on an infinite rough surface from the top, where the spaces above and below the scattering surface are filled with some fixed materials, respectively. The unbounded rough surface-scattering problem is challenging because of three major reasons: (i) an appropriate radiation condition is required as a part of the boundary value problem, since the usual Sommerfeld radiation condition is no longer valid due to the unboundedness of the rough surface; (ii) lack of compactness of the solution functional space due to the unboundedness of the domain; and (iii) computationally, a usual approach is to truncate the open domain into a bounded domain, and thus transparent boundary conditions have to be imposed on the boundary of the truncated bounded domain so that no artificial wave reflection occurs. Despite the large amount of work performed so far for the unbounded rough surface-scattering problems, the aforementioned issues still cannot be considered completely solved, particularly for the transparent boundary conditions from the computational point of view and remain to be the subject matter of much ongoing research. 
This paper consists of two major parts. The first part is concerned with the existence and uniqueness of the solution for a general scattering surface. The second part deals with the derivation of an analytical solution for a special class of scattering surfaces: sufficiently small and smooth deformation of plane surface.

In the first part, the scattering problem is modeled as a boundary value problem for acoustic wave propagation governed by the two-dimensional Helmholtz equation with transparent boundary conditions proposed on plane surfaces confining the scattering surface. Because the usual Sommerfeld radiation condition is not valid, the transparent boundary conditions are derived from the new radiation condition: the total field is consisted of bounded outgoing waves above and below the scattering surface, plus the incident field above the scattering surface. This radiation condition is equivalent to the upward propagating radiation condition proposed for a two-dimensional rough surface-scattering problems by Chandler-Wilde and Zhang [7] and has recently been analyzed carefully by Arens and Hohage [8]. The existence and uniqueness of the weak solution for the model problem are established by using a variational approach. The method enjoys a great generality in the sense that it allows very general surface structures. Throughout we restrict to the case of lossy medium, where the wavenumber is assumed to have a nonzero imaginary part accounting for the energy absorption. We refer to Ritterbusch [9] and Chandler-Wilde and Elschner [10] for related scattering problems where weighted Sobolev spaces are studied for unbounded domains.

The rough surface-scattering problems for the Helmholtz equation have been recently studied by Chandler-Wilde and Monk [11], Chanlder-Wilde et al. [12], and Lechleiter and Ritterbusch [13], who considered variational approaches to solve a two-dimensional or three-dimensional rough surface-scattering problem that models the time-harmonic acoustic wave scattering by a layer of homogeneous or inhomogeneous medium above a sound soft rough surface. We refer to Li et al. [14] of the scattering problem for the vector form of Maxwell's equations with dielectric surfaces, which models the time-harmonic electromagnetic wave by three layers of inhomogeneous medium with two infinite rough surfaces. In addition, the two-dimensional scalar model problem has been considered by integral equation methods in two cases. The first case assumes that the medium is homogeneous and the surface is the graph of a sufficiently smooth bounded function, when the boundary integral equation methods are applicable, for example, Chandler-Wilde et al. [15, 16], Zhang and Chandler-Wilde [17,18], and DeSanto and Martin [19-21]. The second case studied is that the surface is a straight line, e.g., Chandler-Wilde and Zhang [22] and Li [23].

In the second part, we derive an analytical solution for the scattering problem on the basis of a boundary perturbation technique combined with the transformed field expansion, under the assumption that scattering rough surface is a sufficiently small and smooth deformation of a plane surface. By the transformed field expansion, the original problem with complex scattering surface is converted into a set of recursive transmission problems with a flat interface. Furthermore, the nonlocal transparent boundary conditions become local boundary condition in the frequency domain. Therefore, we can reduce the two-dimensional problem into a sequence of onedimensional two-point boundary value problem with exact local boundary conditions. This approach leads not only to the availability of analytical solution in the form of an infinite series but also to an efficient and robust computational method (cf. [24]).

For boundary perturbation methods, we refer to a series of papers by Bruno and Reitich [25-29], Nicholls and Reitich [30], and references cited therein, for the rigorous mathematical and numerical analysis for solving some diffraction grating and obstacle-scattering problems. An improved boundary perturbation algorithm, termed as transformed field expansion, was proposed by Nicholls and Reitich [31], where a change of variables was performed first to flatten the shape of the scattering surface and then followed by the boundary perturbation technique. The transformed field expansion method was shown to be accurate, stable, and robust even at high order, see, for example, Nicholls and Shen [32] and Fang et al. [33] for solving the two-dimensional and three-dimensional bounded obstacle-scattering problems.

We also point out that some related work for the scattering of acoustic and electromagnetic waves in a grating (periodic surface) structure (diffractive optics) and in a cavity (local perturbation of a plane surface). They have been studied extensively by either integral equation methods or variational approaches (cf. Bao [34,35], Ammari et al. [36, 37], Van and Wood [38], Woo [39], and reference therein). More recently, existence of the solution to the acoustic and electromagnetic scattering problem in infinite periodic surface perturbed by a single inhomogeneous object placed inside the periodic structure is established via the integral equation method by Ammari and Bao [40,41]. One may consult Colton and Kress [42, 43], Nédélec [44], and Monk [45] for extensive accounts of the integral equation methods and finite element methods for acoustic and electromagnetic scattering problems.

The outline of this paper is as follows. In Section 2, the model problem is introduced and some regularity properties of the trace operator are discussed. Section 3 is devoted to the derivation of the transparent boundary condition. Some estimates of the capacity operator are introduced. A variational formulation for the unbounded rough surface acoustic scattering problem is introduced by using the transparent boundary condition. The existence and uniqueness of the weak solution for the variational problem are established. With the boundary perturbation technique combined with the transformed field expansion, an analytical solution is derived in Section 4. The paper is concluded with some general remarks and directions for future research in Section 5.

\section{A model problem}

In this section, we shall introduce a mathematical model and define some notation for the scattering problem by an unbounded rough surface. Let the scattering surface be described by the curve $S=\{(x, y): y=f(x), x \in \mathbb{R}\}$ with a bounded and Lipschitz continuous function $f$, as seen in Figure 1. The scattering surface $S$ is embedded in the strip

$$
\Omega=\left\{(x, y) \in \mathbb{R}^{2}: y_{-}<y<y_{+}\right\}=\mathbb{R} \times\left(y_{-}, y_{+}\right),
$$

where $y_{-}$and $y_{+}$are two constants. Let $\Omega_{f}^{+}=\{(x, y): y>f(x)\}$ and $\Omega_{f}^{-}=\{(x, y): y<f(x)\}$ be filled with materials whose wavenumbers are constants $\kappa_{+}$and $\kappa_{-}$, respectively. In fact, the wavenumber satisfies $\kappa_{ \pm}^{2}=\omega^{2} \mu \varepsilon_{ \pm}$, where $\omega$ is the angular frequency, $\mu$ is 


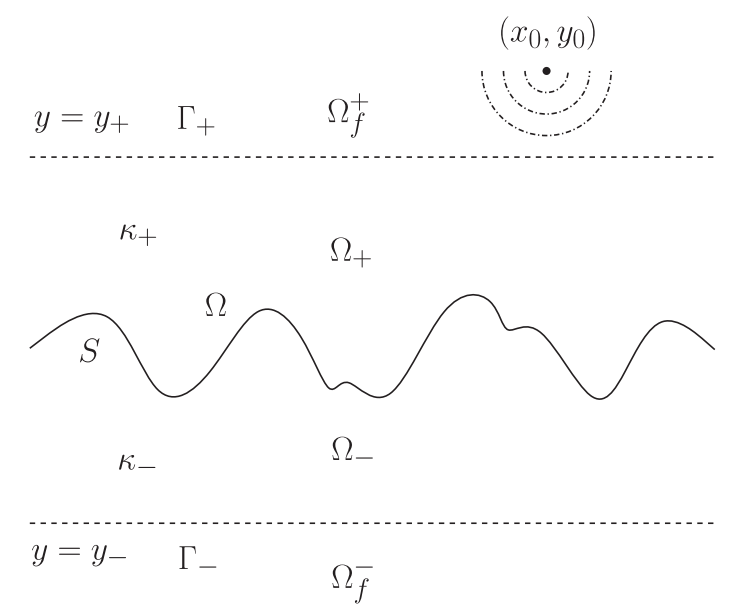

Figure 1. Problem geometry. A wave from the point source at $\left(x_{0}, y_{0}\right)$ is incident on the scattering surface $S$ from the top. The spaces $\Omega_{f}^{+}$(above $S$ ) and $\Omega_{f}^{-}$ (below $S$ ) are filled with materials whose wavenumbers are constants $\kappa_{+}$and $\kappa_{-}$, respectively.

the magnetic permeability that is assumed to be a constant everywhere, and $\varepsilon_{ \pm}$is the electric permittivity in $\Omega_{f}^{ \pm}$. In this work, the wavenumber $\kappa_{ \pm}$is assumed to be a fixed complex number with

$$
\alpha>\operatorname{Re} \kappa_{ \pm}^{2}>0 \text { and } \operatorname{Im} \kappa_{ \pm}^{2}>\beta>0 .
$$

The condition $\operatorname{Im} \kappa_{ \pm}^{2}>0$ accounts for energy absorption. Denote by $\Gamma_{+}=\left\{y=y_{+}\right\}$and $\Gamma_{-}=\left\{y=y_{-}\right\}$the top and bottom boundaries of the domain $\Omega$.

Suppose that a wave generated from a point source is incident on $S$ from the top. Explicitly, the point incident field is taken as the fundamental solution of the Helmholtz equation in $\Omega_{+}$, that is,

$$
u^{\text {inc }}\left(x, y ; x_{0}, y_{0}\right)=\frac{i}{4} H_{0}^{(1)}\left(\kappa+\left|(x, y)-\left(x_{0}, y_{0}\right)\right|\right),
$$

where $H_{0}^{(1)}$ is the Hankel function of first kind with order zero, $\mathbf{x}=(x, y)$ is the observation point, and $\mathbf{x}_{0}=\left(x_{0}, y_{0}\right)$ is a given source point in $\Omega_{+}$. Clearly the incident field satisfies the two-dimensional Helmholtz equation

$$
\Delta u^{\text {inc }}(\mathbf{x})+\kappa_{+}^{2} u^{\text {inc }}(\mathbf{x})=-\delta\left(\mathbf{x}-\mathbf{x}_{0}\right) \quad \text { in } \mathbb{R}^{2},
$$

where $\delta$ is the Dirac delta function.

The scattering of time-harmonic electromagnetic waves in the transverse electric case can also be modeled by the two- dimensional Helmholtz equation:

$$
\Delta u(\mathbf{x})+\kappa^{2} u(\mathbf{x})=-\delta\left(\mathbf{x}-\mathbf{x}_{0}\right) \quad \text { in } \mathbb{R}^{2},
$$

where the wavenumber

$$
\kappa= \begin{cases}\kappa_{+} & \text {in } \Omega_{f}^{+} \\ \kappa_{-} & \text {in } \Omega_{f}^{-}\end{cases}
$$

Because of the unbounded scattering surface, the usual Sommerfeld radiation condition is no longer valid. The radiation condition that we impose is the boundedness of $u$ as $y$ tends to infinity. More precisely, we insist that $u$ is composed of bounded outgoing waves in $\Omega_{+}$and $\Omega_{-}$plus the incident wave $u^{\text {inc }}$ in $\Omega_{+}$.

To describe the boundary value problem and derive its variational formulation, we need to introduce some functional space notations. For $u \in L^{2}\left(\Gamma_{ \pm}\right)$, which is identified with $L^{2}(\mathbb{R})$, we denote by $\hat{u}$ the Fourier transform of $u$ defined by

$$
\hat{u}(\xi)=\int_{\mathbb{R}} u(x) e^{\mathrm{i} x \xi} \mathrm{d} x .
$$

By using Fourier modes, the norm on the space $L^{2}\left(\Gamma_{ \pm}\right)$can be characterized by

$$
\|u\|_{L^{2}\left(\Gamma_{ \pm}\right)}=\|u\|_{L^{2}(\mathbb{R})}=\left[\int_{\mathbb{R}}|u|^{2} \mathrm{~d} x\right]^{1 / 2}=\left[\int_{\mathbb{R}}|\hat{u}|^{2} \mathrm{~d} \xi\right]^{1 / 2} .
$$


We denote by $C_{x}^{\infty}(\Omega)$ the linear space of infinitely differential functions with compact support with respect to the variable $x$ on $\Omega$. Denote by $L^{2}(\Omega)$ the space of complex square integrable functions on $\Omega$ with the norm

$$
\|u\|_{L^{2}(\Omega)}=\left[\int_{y_{-}}^{y_{+}} \int_{\mathbb{R}}|u(x, y)|^{2} \mathrm{~d} x \mathrm{~d} y\right]^{1 / 2}=\left[\int_{y_{-}}^{y_{+}} \int_{\mathbb{R}}|\hat{u}(\xi, y)|^{2} \mathrm{~d} \xi \mathrm{d} y\right]^{1 / 2} .
$$

Denote the Sobolev space: $H^{1}(\Omega)=\left\{D^{s} u \in L^{2}(\Omega)\right.$ for all $\left.|s| \leq 1\right\}$. A simple calculation yields an explicit characterization of the norm in $H^{1}(\Omega)$ via Fourier coefficient:

$$
\|u\|_{H^{1}(\Omega)}^{2}=\int_{y_{-}}^{y_{+}} \int_{\mathbb{R}}\left[\left(1+|\xi|^{2}\right)|\hat{u}(\xi, y)|^{2}+\left|\hat{u}^{\prime}(\xi, y)\right|^{2}\right] \mathrm{d} \xi \mathrm{d} y,
$$

where $\hat{u}^{\prime}(\xi, y)=\partial_{y} \hat{u}(\xi, y)$. In addition, to describe the boundary operator and transparent boundary condition in the formulation of the boundary value problem, we define the trace functional space

$$
H^{s}\left(\Gamma_{ \pm}\right)=\left\{u \in L^{2}\left(\Gamma_{ \pm}\right): \int_{\mathbb{R}}\left(1+|\xi|^{2}\right)^{s}|\hat{u}|^{2} \mathrm{~d} \xi<\infty\right\}
$$

whose norm is characterized by

$$
\|u\|_{H^{s}\left(\Gamma_{ \pm}\right)}^{2}=\int_{\mathbb{R}}\left(1+|\xi|^{2}\right)^{s}|\hat{u}|^{2} \mathrm{~d} \xi
$$

It is clear that the dual space associated with $H^{s}\left(\Gamma_{ \pm}\right)$is the space $H^{-s}\left(\Gamma_{ \pm}\right)$. In particular, it holds the following duality lemma.

Lemma 2.1

The spaces $H^{1 / 2}\left(\Gamma_{ \pm}\right)$and $H^{-1 / 2}\left(\Gamma_{ \pm}\right)$are mutually adjoint with respect to the scalar product in $L^{2}\left(\Gamma_{ \pm}\right)$.

The following lemma is concerned with the density of $C_{x}^{\infty}(\Omega)$. This is important, particularly for our case of unbounded slab $\Omega$ because it allows us to prove results for smooth functions with compact support and extend them by limiting argument to more general functions.

Lemma 2.2

$C_{X}^{\infty}(\Omega)$ is dense in $H^{1}(\Omega)$.

Proof

Noting that $C_{0}^{\infty}\left(\mathbb{R}^{2}\right)$ is dense in $H^{1}\left(\mathbb{R}^{2}\right)$, we have $\left.C_{0}^{\infty}\left(\mathbb{R}^{2}\right)\right|_{\Omega}$ is dense in $\left.H^{1}\left(\mathbb{R}^{2}\right)\right|_{\Omega}$. From the Sobolev extension theorem, $\left.H^{1}\left(\mathbb{R}^{2}\right)\right|_{\Omega}=$ $H^{1}(\Omega)$. Therefore, $\left.C_{x}^{\infty}(\Omega) \supseteq C_{0}^{\infty}\left(\mathbb{R}^{2}\right)\right|_{\Omega}$ is dense in $H^{1}(\Omega)$.

To simply proofs, we shall employ positive constants $C$ and $C_{i}$ as generic constants whose precise values are not required and may change line by line but should always be clear from the context.

The following lemma shows that the space $H^{1 / 2}\left(\Gamma_{ \pm}\right)$and $H^{-1 / 2}\left(\Gamma_{ \pm}\right)$are mutually adjoint under the dual paring $\langle\cdot, \cdot\rangle_{ \pm}$defined by

$$
\langle u, v\rangle_{\Gamma_{ \pm}}=\int_{\mathbb{R}} \hat{u} \overline{\hat{v}} \mathrm{~d} \xi
$$

Note that, from the Parseval formula, the dual paring $\langle u, v\rangle_{ \pm}$is the $L^{2}\left(\Gamma_{ \pm}\right)$inner product between $u$ and $v$ if $u, v \in L^{2}\left(\Gamma_{ \pm}\right)$.

The following trace regularity result in $H^{1 / 2}\left(\Gamma_{ \pm}\right)$is useful in subsequent analysis.

Lemma 2.3

Let $\gamma_{0}=\sqrt{1+\left(y_{+}-y_{-}\right)^{-1}}$. It holds the estimate

$$
\|u\|_{H^{1 / 2}\left(\Gamma_{ \pm}\right)} \leq \gamma_{0}\|u\|_{H^{1}(\Omega)}
$$

for all $u \in H^{1}(\Omega)$.

Proof

First, we have

$$
\begin{aligned}
\left(y_{+}-y_{-}\right)\left|\zeta\left(y_{ \pm}\right)\right|^{2} & =\int_{y_{-}}^{y_{+}}|\zeta(y)|^{2} \mathrm{~d} y+\int_{y_{-}}^{y_{+}} \int_{y}^{y_{ \pm}} \frac{d}{d t}|\zeta(t)|^{2} \mathrm{~d} t \mathrm{~d} y \\
& \leq \int_{y_{-}}^{y_{+}}|\zeta(y)|^{2} \mathrm{~d} y+\left(y_{+}-y_{-}\right) \int_{y_{-}}^{y_{+}} 2|\zeta(y)|\left|\zeta^{\prime}(y)\right| \mathrm{d} y_{1}
\end{aligned}
$$


which implies by the Cauchy-Schwarz inequality that

$$
\left(1+|\xi|^{2}\right)^{1 / 2}\left|\zeta\left(y_{ \pm}\right)\right|^{2} \leq \gamma_{0}^{2}\left(1+|\xi|^{2}\right) \int_{y_{-}}^{y_{+}}|\zeta(y)|^{2} \mathrm{~d} y+\int_{y_{-}}^{y_{+}}\left|\zeta^{\prime}(y)\right|^{2} \mathrm{~d} y
$$

Given $u$ in $H^{1}(\Omega)$, it follows from the definition (2.6) that

$$
\|u\|_{H^{1 / 2}\left(\Gamma_{ \pm}\right)}^{2}=\int_{\mathbb{R}}\left(1+|\xi|^{2}\right)^{1 / 2}\left|\hat{u}\left(\xi, y_{ \pm}\right)\right|^{2} \mathrm{~d} \xi
$$

Using (2.8), we obtain

$$
\begin{aligned}
\left(1+|\xi|^{2}\right)^{1 / 2}\left|\hat{u}\left(\xi, y_{ \pm}\right)\right|^{2} & \leq \gamma_{0}^{2}\left(1+|\xi|^{2}\right) \int_{y_{-}}^{y_{+}}|\hat{u}(\xi, y)|^{2} \mathrm{~d} y+\int_{y_{-}}^{y_{+}}\left|\hat{u}^{\prime}(\xi, y)\right|^{2} \mathrm{~d} y \\
& \leq \gamma_{0}^{2} \int_{y_{-}}^{y_{+}}\left[\left(1+|\xi|^{2}\right)|\hat{u}(\xi, y)|^{2}+\left|\hat{u}^{\prime}(\xi, y)\right|^{2}\right] \mathrm{d} y .
\end{aligned}
$$

The proof is complete by combining the aforementioned estimate and noting the definition of $\|u\|_{H^{1}(\Omega)}$ in (2.5).

\section{Variational problem}

In this section, we shall derive the boundary operators, which map the electric fields to their normal derivatives, introduce the transparent boundary conditions on the artificial boundaries $\Gamma_{ \pm}$, and present a variational formulation for the scattering problem. The existence and uniqueness for the weak solution of the model problem will then be established.

\subsection{Transparent boundary condition}

We wish to reduce problem (2.3) in $\mathbb{R}^{2}$ to the domain $\Omega$. The radiation condition for the scattering problem insists that the total field $u$ is composed of bounded outgoing waves in $\Omega_{+}$and $\Omega_{-}$, plus the incident field $u^{\text {inc }}$ in $\Omega_{+}$. Because the derivative of the transparent boundary conditions on $\Gamma_{+}$and $\Gamma_{-}$are parallel, we will only show how to deduce the transparent boundary condition on $\Gamma_{+}$and state the corresponding transparent boundary condition on $\Gamma_{-}$without derivation.

Observe that the medium is homogeneous above $\Gamma_{+}$. The scattered field $u^{s}=u-u^{\text {inc }}$ satisfies

$$
\Delta u^{s}+\kappa_{+}^{2} u^{s}=0 \quad \text { above } \Gamma_{+} .
$$

By taking Fourier transform of (3.1) with respect to $x$, we have

$$
\frac{\partial^{2} \hat{u}^{s}(\xi, y)}{\partial y^{2}}+\left(\kappa_{+}^{2}-\xi^{2}\right) \hat{u}^{\mathrm{s}}(\xi, y)=0 \quad \text { for } y>y_{+} .
$$

Because only bounded outgoing wave is allowed for the solution of (3.2), we deduce that

$$
\hat{u}^{\mathrm{s}}(\xi, y)=\hat{u}^{\mathrm{s}}\left(\xi, y_{+}\right) e^{\mathrm{i} \beta_{+}\left(y-y_{+}\right)}
$$

where

$$
\beta_{+}^{2}(\xi)=\kappa_{+}^{2}-|\xi|^{2} \quad \text { with } \operatorname{lm} \beta_{+}(\xi)>0
$$

Taking the inverse Fourier transform of $\hat{u}^{\mathrm{s}}(\xi, y)$, we find from (3.3) that

$$
u^{\mathrm{s}}(x, y)=\int_{\mathbb{R}} \hat{u}^{\mathrm{s}}\left(\xi, y_{+}\right) e^{\mathrm{i} \beta_{+}\left(y-y_{+}\right)} e^{\mathrm{i} \xi x} \mathrm{~d} \xi .
$$

Taking the normal derivative, which is the partial derivative with respect to $y$ on $\Gamma_{+}$, and evaluating at $y_{+}$yield

$$
\left.\partial_{\mathbf{n}_{+}} u^{\mathrm{s}}(x, y)\right|_{y=y_{+}}=\int_{\mathbb{R}} \mathrm{i} \beta+\hat{u}^{\mathrm{s}}\left(\xi, y_{+}\right) e^{\mathrm{i} \xi x} \mathrm{~d} \xi
$$

where $\mathbf{n}_{+}$is the unit outward normal on $\Gamma_{+}$, that is, $\mathbf{n}_{+}=(0,1)^{\top}$.

For any given $u$ on $\Gamma_{+}$, define the boundary operator $T_{+}$:

$$
T_{+} u=\int_{\mathbb{R}} \mathrm{i} \beta+\hat{u}\left(\xi, y_{+}\right) e^{\mathrm{i} \xi x} \mathrm{~d} \xi,
$$

which leads to a transparent boundary condition on $\Gamma_{+}$,

$$
\partial_{\mathbf{n}_{+}}\left(u-u^{\text {inc }}\right)=T_{+}\left(u-u^{\text {inc }}\right) .
$$


Equivalently, it can be written as

$$
\partial_{\mathbf{n}_{+}} u=T_{+} u+\rho \text { on } \Gamma_{+},
$$

where

$$
\rho=\partial_{\mathbf{n}_{+}} u^{\text {inc }}-T_{+} u^{\text {inc }} .
$$

Similarly, for any given $u$ on $\Gamma_{-}$, define the boundary operator $T_{-}$:

$$
T_{-} u=\int_{\mathbb{R}} \mathrm{i} \beta-\hat{u}\left(\xi, y_{-}\right) e^{\mathrm{i} \xi x} \mathrm{~d} \xi,
$$

where

$$
\beta_{-}^{2}(\xi)=\kappa_{-}^{2}-|\xi|^{2} \quad \text { with } \operatorname{Im} \beta_{-}(\xi)>0
$$

Thus, we deduce a transparent boundary condition on $\Gamma_{-}$:

$$
\partial_{\mathbf{n}_{-}} u=T_{-} u_{1}
$$

where $\mathbf{n}_{-}$is the unit outward normal on $\Gamma_{-}$, i.e., $\mathbf{n}_{-}=(0,-1)^{\top}$.

To summarize, problem (2.5) with the point incident field given in (2.2) and the radiation condition is reduced to the following problem:

$$
\begin{aligned}
\Delta u+\kappa^{2} u & =0 & & \text { in } \Omega, \\
\partial_{\mathbf{n}_{+}} u & =T_{+} u+\rho & & \text { on } \Gamma_{+}, \\
\partial_{\mathbf{n}_{-}} u & =T_{-} u & & \text { on } \Gamma_{-},
\end{aligned}
$$

with $\kappa$ and $\rho$ given by (2.4) and (3.6), respectively.

\section{Remark 3.1}

Clearly, transparent boundary conditions (3.5) and (3.7) are nonlocal in the physical domain. However, they are local boundary conditions in the frequency domain. In fact, taking the Fourier transform of (3.5) and (3.7) on both sides, we have

$$
\partial_{\mathbf{n}_{ \pm}} \hat{u}\left(\xi, y_{ \pm}\right)=\widehat{T_{ \pm} u}=\mathrm{i} \beta_{ \pm}(\xi) \hat{u}\left(\xi, y_{ \pm}\right)
$$

This observation is critical and makes it possible to derive an analytical solution in Section 4.

To derive some estimates on the boundary operators, it is useful to introduce the following notations. Define

$$
\kappa_{ \pm}^{2}=\varphi_{ \pm}+\mathrm{i} \psi_{ \pm}
$$

where

$$
\varphi_{ \pm}=\operatorname{Re} \kappa_{ \pm}^{2} \quad \text { and } \quad \psi_{ \pm}=\operatorname{Im} \kappa_{ \pm}^{2} .
$$

Denote by

$$
\beta_{ \pm}^{2}=\kappa_{ \pm}^{2}-|\xi|^{2}=\phi_{ \pm}+\mathrm{i} \psi_{ \pm},
$$

where

$$
\phi_{ \pm}=\operatorname{Re} \kappa_{ \pm}^{2}-|\xi|^{2}=\varphi_{ \pm}-|\xi|^{2}
$$

A simple calculation gives

$$
\beta_{ \pm}=a_{ \pm}+\mathrm{i} b_{ \pm}
$$

where

$$
a_{ \pm}=\operatorname{Re} \beta_{ \pm}=\left(\frac{\sqrt{\phi_{ \pm}^{2}+\psi_{ \pm}^{2}}+\left|\phi_{ \pm}\right|}{2}\right)^{1 / 2} \quad \text { and } \quad b_{ \pm}=\operatorname{Im} \beta_{ \pm}=\left(\frac{\sqrt{\phi_{ \pm}^{2}+\psi_{ \pm}^{2}}-\left|\phi_{ \pm}\right|}{2}\right)^{1 / 2}
$$

It follows from (3.11) that

$$
\varphi_{ \pm} \geq \phi_{ \pm}>-\infty \quad \text { and } \quad\left|\phi_{ \pm}\right| \geq 0
$$


which yields

$$
a_{ \pm} \geq\left(\frac{\operatorname{Im} \kappa_{ \pm}^{2}}{2}\right)^{1 / 2} \text { and }\left(\frac{\operatorname{Im} \kappa_{ \pm}^{2}}{2}\right)^{1 / 2} \geq b_{ \pm}>0
$$

Lemma 3.1

The boundary operator $T_{ \pm}: H^{1 / 2}\left(\Gamma_{ \pm}\right) \rightarrow H^{-1 / 2}\left(\Gamma_{ \pm}\right)$is continuous.

Proof

For any $u, v \in H^{1 / 2}\left(\Gamma_{ \pm}\right)$, it follows from definitions (2.7), (3.5), and (3.7) that

$$
\left\langle T_{ \pm} u, v\right\rangle=\mathrm{i} \int_{\mathbb{R}} \beta_{ \pm} \hat{u} \overline{\hat{v}} \mathrm{~d} \xi=\mathrm{i} \int_{\mathbb{R}} \beta_{ \pm}\left(1+|\xi|^{2}\right)^{-1 / 2} \times\left(1+|\xi|^{2}\right)^{1 / 4} \hat{u} \times\left(1+|\xi|^{2}\right)^{1 / 4} \hat{v} \mathrm{~d} \xi
$$

To prove the lemma, it is required to estimate

$$
\frac{\left|\beta_{ \pm}\right|}{\left(1+|\xi|^{2}\right)^{1 / 2}}=\left[\frac{\psi_{ \pm}^{2}+\phi_{ \pm}^{2}}{\left(1+\varphi_{ \pm}-\phi_{ \pm}\right)^{2}}\right]^{1 / 4}
$$

Let

$$
F_{ \pm}(t)=\frac{\psi_{ \pm}^{2}+t^{2}}{\left(1+\varphi_{ \pm}-t\right)^{2}}, \quad-\infty<t \leq \varphi_{ \pm}
$$

It can be verified that $F_{ \pm}(t)$ decreases for $-\infty<t \leq K_{ \pm}=-\psi_{ \pm}^{2} /\left(1+\varphi_{ \pm}\right)$and increases for $K_{ \pm} \leq t \leq \varphi_{ \pm}$. Hence, a simple calculation yields

$$
F_{ \pm}(t) \leq \max \left\{F_{ \pm}(-\infty), F_{ \pm}\left(\varphi_{ \pm}\right)\right\}=\max \left\{1,\left|\kappa_{ \pm}\right|^{4}\right\}
$$

Combining the aforementioned estimates yields

$$
\left|\left\langle T_{ \pm} u, v\right\rangle\right| \leq \gamma_{ \pm}\|u\|_{H^{1 / 2}\left(\Gamma_{ \pm}\right)}\|v\|_{H^{1 / 2}\left(\Gamma_{ \pm}\right)^{\prime}}
$$

where

$$
\gamma_{ \pm}=\max \left\{1,\left|\kappa_{ \pm}\right|\right\}
$$

Thus, from Lemma 2.2, we have

$$
\left\|T_{ \pm} u\right\|_{H^{-1 / 2}\left(\Gamma_{ \pm}\right)} \leq \sup _{v \in H^{1 / 2}\left(\Gamma_{ \pm}\right)} \frac{\left|\left\langle T_{ \pm} u, v\right\rangle\right|}{\|v\|_{H^{1 / 2}\left(\Gamma_{ \pm}\right)}} \leq \gamma_{ \pm}\|u\|_{H^{1 / 2}\left(\Gamma_{ \pm}\right)} .
$$

Lemma 3.2

Let $u \in H^{1 / 2}\left(\Gamma_{ \pm}\right)$. It holds that $\operatorname{Re}\left\langle T_{ \pm} u, u\right\rangle \leq 0$ and $\operatorname{Im}\left\langle T_{ \pm} u, u\right\rangle \geq 0$. If $\operatorname{Re}\left\langle T_{ \pm} u, u\right\rangle=0$ or $\operatorname{Im}\left\langle T_{ \pm} u, u\right\rangle=0$, then $u=0$.

Proof

By definitions (2.7), (3.5), (3.7), and (3.12), we find

$$
\left\langle T_{ \pm} u, u\right\rangle=\mathrm{i} \int_{\mathbb{R}} \beta_{ \pm}|\hat{u}|^{2} \mathrm{~d} \xi=\mathrm{i} \int_{\mathbb{R}} a_{ \pm}|\hat{u}|^{2} \mathrm{~d} \xi-\int_{\mathbb{R}} b_{ \pm}|\hat{u}|^{2} \mathrm{~d} \xi
$$

Taking the real part gives

$$
\operatorname{Re}\left\langle T_{ \pm} u, u\right\rangle=-\int_{\mathbb{R}} b_{ \pm}|\hat{u}|^{2} \mathrm{~d} \xi \leq 0
$$

and taking the imaginary part yields

$$
\operatorname{Im}\left\langle T_{ \pm} u, u\right\rangle=\int_{\mathbb{R}} a_{ \pm}|\hat{u}|^{2} \mathrm{~d} \xi \geq 0
$$

Furthermore, $\operatorname{Re}\left\langle T_{ \pm} u, u\right\rangle=0$ implies that $\hat{u}=0$, and $\operatorname{Im}\left\langle T_{ \pm} u, u\right\rangle=0$ implies that $\hat{u}=0$. Hence, either $\operatorname{Re}\left\langle T_{ \pm} u, u\right\rangle=0$ implies $u=0$ or $\operatorname{Im}\left\langle T_{ \pm} u, u\right\rangle=0$ implies $u=0$. 


\subsection{Well-posedness}

We now present a variational formulation of Helmholtz equation (3.8) in $H^{1}(\Omega)$ and give a simple proof of the well-posedness for the boundary value problem.

Multiplying (3.8) by the complex conjugate of a test function $v \in H^{1}(\Omega)$, integrating over $\Omega$, and using integration by parts (recall that Lemma 2.2 implies that the lateral boundary integrals vanish as $|x| \rightarrow \infty)$, we deduce the variational formulation for scattering problem (3.8): find $u \in H^{1}(\Omega)$ such that

$$
a(u, v)=\langle\rho, v\rangle_{\Gamma_{+}} \quad \text { for all } v \in H^{1}(\Omega),
$$

with the sesquilinear form

$$
a(u, v)=\int_{\Omega}\left(\nabla u \cdot \nabla \bar{v}-\kappa^{2} u \bar{v}\right)-\int_{\Gamma_{+}} T_{+} u \bar{v}-\int_{\Gamma_{-}} T_{-} u \bar{v}
$$

and the linear functional

$$
\langle\rho, v\rangle_{+}=\int_{\Gamma_{+}} \rho \bar{v}
$$

Lemma 3.3

Under condition (2.1), we have $\rho \in H^{s}\left(\Gamma_{+}\right)$for all $s \in \mathbb{R}$.

Proof

Recall

$$
\rho\left(x, y ; x_{0}, y_{0}\right)=\partial_{\mathbf{n}_{+}} u^{\text {inc }}\left(x, y ; x_{0}, y_{0}\right)-T_{+} u^{\text {inc }}\left(x, y ; x_{0}, y_{0}\right) .
$$

It suffices to prove

$$
\left\|\rho\left(x, y_{+} ; x_{0}, y_{0}\right)\right\|_{H^{s}\left(\Gamma_{+}\right)}^{2}=\int_{\mathbb{R}}\left(1+|\xi|^{2}\right)^{s}\left|\hat{\rho}\left(\xi, y_{+} ; x_{0}, y_{0}\right)\right|^{2} \mathrm{~d} \xi<\infty
$$

Let

$$
\hat{\rho}^{\text {inc }}\left(\xi, y ; x_{0}, y_{0}\right)=\int_{\mathbb{R}} u^{\text {inc }}\left(x, y ; x_{0}, y_{0}\right) e^{-\mathrm{i} \xi x} \mathrm{~d} x
$$

be the Fourier transform of $u^{\text {inc }}\left(x, y ; x_{0}, y_{0}\right)$ with respect to $x$. Because the incident field is taken as the fundamental solution for the Helmholtz equation with number $\kappa_{+}$, it can be explicitly written as

$$
\hat{\rho}^{\text {inc }}\left(\xi, y ; x_{0}, y_{0}\right)=\frac{i}{2 \beta_{+}(\xi)} e^{i \beta+\left|y-y_{0}\right|} e^{-i \xi x_{0}}
$$

Noticing $y_{0}>y_{+}$, we have by simple calculations that

$$
\left.\partial_{\mathbf{n}_{+}} \hat{u}^{\mathrm{inc}}\left(x, y ; x_{0}, y_{0}\right)\right|_{y=y_{+}}=-\frac{1}{2} e^{\mathrm{i} \beta_{+}\left(y_{0}-y_{+}\right)} e^{-\mathrm{i} \xi x_{0}} .
$$

Using the definition of the boundary operator $T_{+}$, we have

$$
T_{+} u^{\text {inc }}\left(x, y_{+} ; x_{0}, y_{0}\right)=\int_{\mathbb{R}} \mathrm{i} \beta_{+}(\xi) \hat{u}^{\text {inc }}\left(\xi, y_{+} ; x_{0}, y_{0}\right) e^{\mathrm{i} \xi x} \mathrm{~d} \xi,
$$

which gives after taking the Fourier transform

$$
\widehat{T_{+} u^{\mathrm{inc}}}\left(\xi, y_{+} ; x_{0}, y_{0}\right)=\mathrm{i} \beta_{+}(\xi) \hat{u}^{\mathrm{inc}}\left(\xi, y_{+} ; x_{0}, y_{0}\right)=-\frac{1}{2} e^{\mathrm{i} \beta_{+}\left(y_{0}-y_{+}\right)} e^{-\mathrm{i} \xi x_{0}}
$$

Combining (3.17), (3.18), and (3.19), we obtain after plugging (3.12) that

$$
\hat{\rho}\left(\xi, y_{+} ; x_{0}, y_{0}\right)=-e^{i \beta_{+}\left(y_{0}-y_{+}\right)} e^{-i \xi x_{0}}=-e^{i a_{+}(\xi)\left(y_{0}-y_{+}\right)} e^{-b_{+}(\xi)\left(y_{0}-y_{+}\right)} e^{-i \xi x_{0}}
$$

which gives

$$
\left|\hat{\rho}\left(\xi, y_{+} ; x_{0}, y_{0}\right)\right|^{2}=e^{-2 b_{+}(\xi)\left(y_{0}-y_{+}\right)} .
$$


Using (3.11) and (3.12) yields

$$
b_{+}(\xi)=\left(\frac{\sqrt{\left(|\xi|^{2}-\varphi_{+}\right)^{2}+\psi_{+}^{2}}+\left.|| \xi\right|^{2}-\varphi_{+} \mid}{2}\right)^{1 / 2}
$$

where $\varphi_{+}=\operatorname{Re} \kappa_{+}^{2}$ and $\psi_{+}=\operatorname{Im} \kappa_{+}^{2}$ are fixed constants. It is easy to check that $b_{+}(\xi) \sim|\xi|$ as $|\xi| \rightarrow \infty$. Hence, we deduce that

$$
\begin{aligned}
\left\|\rho\left(x, y_{+} ; x_{0}, y_{0}\right)\right\|_{H^{s}\left(\Gamma_{+}\right)}^{2} & =\int_{\mathbb{R}}\left(1+|\xi|^{2}\right)^{s}\left|\hat{\rho}\left(\xi, y_{+} ; x_{0}, y_{0}\right)\right|^{2} \mathrm{~d} \xi \\
& =\int_{\mathbb{R}}\left(1+|\xi|^{2}\right)^{s} e^{-2 b_{+}(\xi)\left(y_{0}-y_{+}\right)} \mathrm{d} \xi<\infty
\end{aligned}
$$

for all $s \in \mathbb{R}$, which completes the proof.

\section{Remark 3.2}

If the incident field is a plane wave $u^{\mathrm{inc}}(x, y)=e^{\mathrm{i} \alpha x-\mathrm{i} \beta y}$, where $\alpha=\kappa_{+} \sin \theta, \beta=\kappa_{+} \cos \theta$, and $\theta$ is the angel of incidence, then linear functional (3.16) is not well defined. In fact, a simple calculation yields

$$
\rho(x)=-2 \mathrm{i} \beta e^{\mathrm{i} \alpha x-\mathrm{i} \beta y+}=-2 \mathrm{i} \beta e^{-\mathrm{i} \beta y}+e^{\mathrm{i} \operatorname{Re} \kappa+\sin \theta x} e^{-\operatorname{Im} \kappa+\sin \theta x} .
$$

Thus, it can be easily verified that $\|\rho\|_{L^{2}\left(\Gamma_{+}\right)}=\infty$, that is, $\rho \notin L^{2}\left(\Gamma_{+}\right)$. This is the reason why we consider the incident field generated from a point source instead of the plane wave.

Theorem 3.1

Under condition (2.1), variational problem (3.14) has a unique solution in $H^{1}(\Omega)$ satisfying

$$
\|u\|_{H^{1}(\Omega)} \leq\left(1+\frac{1+\alpha}{\beta}\right)\left\|\partial_{\mathbf{n}_{+}} u^{\mathrm{inc}}-T_{+} u^{\mathrm{inc}}\right\|_{H^{-1 / 2}\left(\Gamma_{+}\right)} .
$$

Proof

It suffices to prove the continuity and coercivity of the sesquilinear form $a$. The continuity follows directly from the Cauchy-Schwarz inequality, Lemma 3.1, and Lemma 2.3:

$$
\begin{aligned}
|a(u, v)| \leq & C_{1}\|u\|_{H^{1}(\Omega)}\|v\|_{H^{1}(\Omega)}+C_{2}\left\|T_{+} u\right\|_{H^{-1 / 2}\left(\Gamma_{+}\right)}\|v\|_{H^{1 / 2}\left(\Gamma_{+}\right)} \\
& +C_{3}\left\|T_{-} u\right\|_{H^{-1 / 2}\left(\Gamma_{-}\right)}\|v\|_{H^{1 / 2}\left(\Gamma_{-}\right)} \\
\leq & C_{1}\|u\|_{H^{1}(\Omega)}\|v\|_{H^{1}(\Omega)}+C_{2}\|u\|_{H^{1 / 2}\left(\Gamma_{+}\right)}\|v\|_{H^{1 / 2}\left(\Gamma_{+}\right)} \\
& +C_{3}\|u\|_{H^{1 / 2}\left(\Gamma_{-}\right)}\|v\|_{H^{1 / 2}\left(\Gamma_{-}\right)} \\
\leq & C\|u\|_{H^{1}(\Omega)}\|v\|_{H^{1}(\Omega)} .
\end{aligned}
$$

Taking the real part of the sesquilinear form $a$ and using Lemma 3.2 yield

$$
\begin{aligned}
\operatorname{Re}[a(u, u)] & =\int_{\Omega}|\nabla u|^{2}-\int_{\Omega} \operatorname{Re}\left(\kappa^{2}\right)|u|^{2}-\operatorname{Re}\left\langle T_{+} u, u\right\rangle_{\Gamma_{+}}-\operatorname{Re}\left\langle T_{-} u, u\right\rangle_{\Gamma_{-}} \\
& \geq \int_{\Omega}|\nabla u|^{2}-\int_{\Omega} \operatorname{Re}\left(\kappa^{2}\right)|u|^{2} \geq\|\nabla u\|_{L^{2}(\Omega)}^{2}-\alpha\|u\|_{L^{2}(\Omega)}^{2} .
\end{aligned}
$$

Taking the imaginary part of the sesquilinear form $a$ and using Lemma 3.2 give

$$
\operatorname{Im}[a(u, u)]=-\int_{\Omega} \operatorname{Im}\left(\kappa^{2}\right)|u|^{2}-\operatorname{Im}\left\langle T_{+} u, u\right\rangle_{\Gamma_{+}}-\operatorname{Im}\left\langle T_{-} u, u\right\rangle_{\Gamma_{-}} \leq-\int_{\Omega} \operatorname{Im}\left(\kappa^{2}\right)|u|^{2},
$$

which, along with (2.1), leads to

$$
|\operatorname{Im}[a(u, u)]| \geq \beta\|u\|_{L^{2}(\Omega)}^{2} .
$$

Denote $\gamma=(1+\alpha) / \beta>0$. It follows from

$$
|a(u, u)| \geq|\operatorname{Re}[a(u, u)]| \quad \text { and } \quad \gamma|a(u, u)| \geq \gamma|\operatorname{Im}[a(u, u)]|
$$

that we have

$$
\begin{aligned}
(1+\gamma)|a(u, u)| & \geq|\operatorname{Re}[a(u, u)]|+\gamma|\operatorname{Im}[a(u, u)]| \\
& \geq\|\nabla u\|_{L^{2}(\Omega)}^{2}-\alpha\|u\|_{L^{2}(\Omega)}^{2}+\gamma \beta\|u\|_{L^{2}(\Omega)}^{2} \geq\|u\|_{H^{1}(\Omega)}^{2} .
\end{aligned}
$$


It follows from the Lax-Milgram lemma and Lemma 3.3 that there exists a unique weak solution of variational problem (3.14) in $H^{1}(\Omega)$ satisfying (3.20).

Remark 3.3

Classical results from the theory of elliptic partial differential equations in Gilbarg and Trundinger [46] indicate that if $\rho=\partial_{\mathbf{n}_{+}} u^{\text {inc }}-$ $T_{+} u^{\text {inc }} \in H^{1 / 2}\left(\Gamma_{+}\right)$, then the unique solution satisfies

$$
\|u\|_{H^{2}(\Omega)} \leq C\left\|\partial_{\mathbf{n}_{+}} u^{\text {inc }}-T_{+} u^{\text {inc }}\right\|_{H^{1 / 2}\left(\Gamma_{+}\right)^{\prime}}
$$

where $C$ is a positive constant.

\section{Boundary perturbation method}

In this section, we study the method of boundary perturbation and deduce an analytical solution for the unbounded rough surfacescattering problem. Here, the scattering profile $f$ is assumed to a bounded and twice continuously differentiable function. First, the scattering problem is formulated as a transmission problem where the scattering profile plays the role of the interface. Then, the transformed field expansion method (cf. [31]) is applied to derive the analytical solution as an infinite series.

\subsection{Transmission problem}

We formulate variational problem (3.14) into an equivalent transmission problem or interface problem, which is of more convenient form for the method of boundary perturbation.

Denote $\Omega_{+}=\Omega_{f}^{+} \cap \Omega$ and $\Omega_{-}=\Omega_{f}^{-} \cap \Omega$, as seen in Figure 1. Consider Helmholtz equation (2.3) in $\Omega_{+}$and $\Omega_{-}$, respectively:

$$
\begin{aligned}
& \Delta u^{+}+\kappa_{+}^{2} u^{+}=0 \quad \text { in } \Omega_{+}, \\
& \Delta u^{-}+\kappa_{-}^{2} u^{-}=0 \quad \text { in } \Omega_{-} .
\end{aligned}
$$

Recall nonlocal transparent boundary conditions (3.5) and (3.7):

$$
\begin{gathered}
\partial_{\mathbf{n}_{+}} u^{+}=T_{+} u^{+}+\rho \quad \text { on } \Gamma_{+}, \\
\partial_{\mathbf{n}_{-}} u^{-}=T_{-} u^{-} \quad \text { on } \Gamma_{-} .
\end{gathered}
$$

Following from the jump conditions, we obtain that the field and its normal derivative are continuous across the scattering surface $S$, that is,

$$
\begin{gathered}
u^{+}(x, f(x))=u^{-}(x, f(x)), \\
\partial_{\mathbf{n}} u^{+}(x, f(x))=\partial_{\mathbf{n}} u^{-}(x, f(x)),
\end{gathered}
$$

where $\mathbf{n}=\left(n_{1}, n_{2}\right)^{\top}$ is the unit normal vector pointing from $\Omega_{+}$to $\Omega_{-}$. Explicitly, we have

$$
n_{1}=\frac{f^{\prime}(x)}{\sqrt{1+\left[f^{\prime}(x)\right]^{2}}} \quad \text { and } \quad n_{2}=-\frac{1}{\sqrt{1+\left[f^{\prime}(x)\right]^{2}}} \text {. }
$$

Hence, the transmission problem is to find the fields $u^{+}$and $u^{-}$, which satisfy Helmholtz equations (4.1) and (4.2), continuity conditions (4.5) and (4.6), and boundary conditions (4.3) and (4.4).

\section{Remark 4.1}

It is easy to see that the solution $u$ of variational problem (3.14) restricted to $\Omega_{+}$and $\Omega_{-}$, that is, $u^{+}=\left.u\right|_{\Omega_{+}}$and $u^{-}=\left.u\right|_{\Omega_{-}}$, is the solution of transmission problem (4.1)-(4.6). Conversely, the solution $u^{+}$and $u^{-}$of transmission problem (4.1)-(4.6) constructs the solution of variational problem (3.14) in the domain $\Omega$.

\subsection{Transformed field expansion}

The transformed field expansion method, as applied to the unbounded rough surface scattering, begins with the change of variables

$$
x_{1}=x, \quad y_{1}=y_{+}\left(\frac{y-f}{y_{+}-f}\right), \quad f<y<y_{+},
$$


and

$$
x_{2}=x, \quad y_{2}=y_{-}\left(\frac{y-f}{y_{-}-f}\right), \quad y_{-}<y<f,
$$

which maps the perturbed domains $\Omega_{+}$and $\Omega_{-}$to unperturbed strip domains $D_{+}$and $D_{-}$, respectively. We now seek to restate transmission problem (4.1)-(4.6) in these transformed coordinates. It is easy to verify the differentiation rules

$$
\begin{aligned}
& \partial_{x}=\partial_{x_{1}}-f^{\prime}\left(\frac{y_{+}-y_{1}}{y_{+}-f}\right) \partial_{y_{1}}, \\
& \partial_{y}=\left(\frac{y_{+}}{y_{+}-f}\right) \partial_{y_{1}},
\end{aligned}
$$

for $f<y<y_{+}$, and

$$
\begin{aligned}
& \partial_{x}=\partial_{x_{2}}-f^{\prime}\left(\frac{y_{-}-y_{2}}{y_{-}-f}\right) \partial_{y_{2}} \\
& \partial_{y}=\left(\frac{y_{-}}{y_{-}-f}\right) \partial_{y_{2}}
\end{aligned}
$$

for $y_{-}<y<f$.

Introduce new functions $w^{+}\left(x_{1}, y_{1}\right)=u^{+}(x, y)$ and $w^{-}\left(x_{2}, y_{2}\right)=u^{-}(x, y)$ under the transformation. It can be verified after tedious but straightforward calculations that $w^{+}$and $w^{-}$, upon dropping the subscript, satisfy the equation

$$
\begin{gathered}
c_{1}^{+} \frac{\partial^{2} w^{+}}{\partial x^{2}}+c_{2}^{+} \frac{\partial^{2} w^{+}}{\partial y^{2}}+c_{3}^{+} \frac{\partial^{2} w^{+}}{\partial x \partial y}+c_{4}^{+} \frac{\partial w^{+}}{\partial y}+c_{1}^{+} \kappa_{+}^{2} w^{+}=0, \quad 0<y<y_{+}, \\
c_{1}^{-} \frac{\partial^{2} w^{-}}{\partial x^{2}}+c_{2}^{-} \frac{\partial^{2} w^{-}}{\partial y^{2}}+c_{3}^{-} \frac{\partial^{2} w^{-}}{\partial x \partial y}+c_{4}^{-} \frac{\partial w^{-}}{\partial y}+c_{1}^{-} \kappa_{-}^{2} w^{-}=0, \quad y_{-}<y<0,
\end{gathered}
$$

where

$$
\begin{aligned}
& c_{1}^{ \pm}=\left(y_{ \pm}-f\right)^{2} \\
& c_{2}^{ \pm}=\left[f^{\prime}\left(y_{ \pm}-y\right)\right]^{2}+y_{+}^{2} \\
& c_{3}^{ \pm}=-2 f^{\prime}\left(y_{ \pm}-y\right)\left(y_{ \pm}-f\right) \\
& c_{4}^{ \pm}=-\left(y_{ \pm}-y\right)\left[f^{\prime \prime}\left(y_{ \pm}-f\right)+2\left(f^{\prime}\right)^{2}\right] .
\end{aligned}
$$

The nonlocal transparent boundary conditions are

$$
\begin{gathered}
\partial_{\mathbf{n}_{+}} w^{+}=\partial_{y} w^{+}=\left(1-\frac{f}{y_{+}}\right)\left(T_{+} w^{+}+\rho\right), \quad y=y_{+}, \\
\partial_{\mathbf{n}_{-}} w^{-}=-\partial_{y} w^{-}=\left(1-\frac{f}{y_{-}}\right) T_{-} w^{-}, \quad y=y_{-} .
\end{gathered}
$$

The continuity conditions at the interface $y=0$ reduce to

$$
\begin{aligned}
w^{+}(x, 0) & =w^{-}(x, 0) \\
\left(\frac{y_{+}}{y_{+}-f}\right) \partial_{y} w^{+}(x, 0) & =\left(\frac{y_{-}}{y_{-}-f}\right) \partial_{y} w^{-}(x, 0) .
\end{aligned}
$$

Now, we use a classical boundary perturbation argument. Let $f=\varepsilon g$ with $\varepsilon$ sufficiently small, and consider the formal expansions of $\left(w^{+}, w^{-}\right)$in a power series of $\varepsilon$ :

$$
w^{+}(x, y ; \varepsilon)=\sum_{n=0}^{\infty} w_{n}^{+}(x, y) \varepsilon^{n} \quad \text { and } \quad w^{-}(x, y ; \varepsilon)=\sum_{n=0}^{\infty} w_{n}^{-}(x, y) \varepsilon^{n} .
$$

Substituting $f=\varepsilon g$ into $c_{j}^{ \pm}$and inserting the aforementioned expansions into (4.7) and (4.8), we may derive the recursions for $w_{n}^{+}$ and $w_{n}^{-}$:

$$
\frac{\partial^{2} w_{n}^{+}}{\partial x^{2}}+\frac{\partial^{2} w_{n}^{+}}{\partial y^{2}}+\kappa_{+}^{2} w_{n}^{+}=v_{n}^{+}, \quad 0<y<y_{+}
$$




$$
\frac{\partial^{2} w_{n}^{-}}{\partial x^{2}}+\frac{\partial^{2} w_{n}^{-}}{\partial y^{2}}+\kappa_{-}^{2} w_{n}^{+}=v_{n}^{-}, \quad y_{-}<y<0,
$$

where

$$
\begin{aligned}
v_{n}^{ \pm}= & \frac{2 g}{y_{ \pm}} \frac{\partial^{2} w_{n-1}^{ \pm}}{\partial x^{2}}+\frac{2 g^{\prime}\left(y_{ \pm}-y\right)}{y_{ \pm}} \frac{\partial^{2} w_{n-1}^{ \pm}}{\partial x \partial y}+\frac{g^{\prime \prime}\left(y_{ \pm}-y\right)}{y_{ \pm}} \frac{\partial w_{n-1}^{ \pm}}{\partial y}+\frac{2 \kappa_{ \pm}^{2} g}{y_{ \pm}} w_{n-1}^{ \pm} \\
& -\frac{g^{2}}{y_{ \pm}^{2}} \frac{\partial^{2} w_{n-2}^{ \pm}}{\partial x^{2}}-\frac{\left(g^{\prime}\right)^{2}\left(y_{ \pm}-y\right)^{2}}{y_{ \pm}^{2}} \frac{\partial^{2} w_{n-2}^{ \pm}}{\partial y^{2}}-\frac{2 g g^{\prime}\left(y_{ \pm}-y\right)}{y_{ \pm}^{2}} \frac{\partial^{2} w_{n-2}^{ \pm}}{\partial x \partial y} \\
& +\frac{\left[2\left(g^{\prime}\right)^{2}-g g^{\prime \prime}\right]\left(y_{ \pm}-y\right)}{y_{ \pm}^{2}} \frac{\partial w_{n-2}^{ \pm}}{\partial y}-\frac{\kappa_{ \pm}^{2} g^{2}}{y_{ \pm}^{2}} w_{n-2}^{ \pm} .
\end{aligned}
$$

Nonlocal boundary conditions (4.9) and (4.10) become

$$
\begin{aligned}
& \partial_{y} w_{n}^{+}-T_{+} w_{n}^{+}=\rho_{n}^{+}, \quad y=y_{+}, \\
& \partial_{y} w_{n}^{-}+T_{-} w_{n}^{-}=\rho_{n}^{-}, \quad y=y_{-},
\end{aligned}
$$

where

$$
\begin{aligned}
& \rho_{0}^{+}=\rho, \quad \rho_{1}^{+}=-\left(\frac{g}{y_{+}}\right) T_{+} w_{0}^{+}-\left(\frac{g}{y_{+}}\right) \rho, \quad \rho_{n}^{+}=-\left(\frac{g}{y_{+}}\right) T_{+} w_{n-1}^{+}, \quad n=2,3, \ldots, \\
& \rho_{0}^{-}=0, \quad \rho_{n}^{-}=\left(\frac{g}{y_{-}}\right) T_{-} w_{n-1}^{-}, \quad n=1,2, \ldots
\end{aligned}
$$

The continuity conditions at the interface $y=0$ reduce to

$$
\begin{gathered}
w_{n}^{+}(x, 0)-w_{n}^{-}(x, 0)=0, \\
\partial_{y} w_{n}^{+}(x, 0)-\partial_{y} w_{n}^{-}(x, 0)=\varrho_{n},
\end{gathered}
$$

where

$$
\varrho_{0}=0, \quad \varrho_{n}=\left(\frac{g}{y_{-}}\right) \partial_{y} w_{n-1}^{+}-\left(\frac{g}{y_{+}}\right) \partial_{y} w_{n-1}^{-}, \quad n=1,2, \ldots .
$$

Note that Helmholtz problem (4.14)-(4.15) for the current terms $w_{n}^{ \pm}$involve some nonhomogeneous terms $v_{n}^{ \pm}, \rho_{n}^{ \pm}$, and $\varrho_{n}$, which only depend on previous two terms $w_{n-1}^{ \pm}$and $w_{n-2}^{ \pm}$. Thus, transmission problems (4.14)-(4.19) indeed can be solved in a recursive manner starting from $n=0$.

Taking the Fourier transform of (4.14) and (4.15) with respect to the variable $x$ yields

$$
\begin{gathered}
\frac{\partial^{2} \hat{w}_{n}^{+}}{\partial y^{2}}+\left(\kappa_{+}^{2}-\xi^{2}\right) \hat{w}_{n}^{+}=\hat{v}_{n}^{+}, \quad 0<y<y_{+}, \\
\frac{\partial^{2} \hat{w}_{n}^{-}}{\partial y^{2}}+\left(\kappa_{-}^{2}-\xi^{2}\right) \hat{w}_{n}^{-}=\hat{v}_{n}^{-}, \quad y_{-}<y<0 .
\end{gathered}
$$

According to Remark 3.1, the nonlocal boundary conditions become local boundary condition in the frequency domain:

$$
\begin{gathered}
\partial_{y} \hat{w}_{n}^{+}-\mathrm{i} \beta_{+} \hat{w}_{n}^{+}=\hat{\rho}_{n}^{+}, \quad y=y_{+}, \\
\partial_{y} \hat{w}_{n}^{-}+\mathrm{i} \beta_{-} \hat{w}_{n}^{-}=\hat{\rho}_{n}^{-}, \quad y=y_{-} .
\end{gathered}
$$

The continuity conditions reduce to

$$
\begin{gathered}
\hat{w}_{n}^{+}(\xi, 0)-\hat{w}_{n}^{-}(\xi, 0)=0, \\
\partial_{y} \hat{w}_{n}^{+}(\xi, 0)-\partial_{y} \hat{w}_{n}^{-}(\xi, 0)=\hat{\varrho}_{n} .
\end{gathered}
$$

After taking the Fourier transform, two-dimensional problem (4.14)-(4.19) reduces to one-dimensional transmission problem (4.20)-(4.25) in the frequency domain. This problem falls in the class of the so-called two-point boundary value problem, whose detailed 
solution is discussed in Appendix B. An application of Theorem B.1, along with (B.13) and (B.23), leads to the following explicit solution of the transmission problem.

Theorem 4.1

Let $f$ be in $C^{2}(\mathbb{R})$, and assume that $\left(w^{+}, w^{-}\right)$admit the asymptotic expansions (4.13). Then the fields $\left(w_{n}^{+}, w_{n}^{-}\right)$are determined as the unique solution of the transmission problem (4.20)-(4.25), given explicitly as

$$
\begin{aligned}
& \hat{w}_{n}^{+}(\xi, y)=e^{\mathrm{i} \beta+y} \hat{w}_{n}^{+}(\xi, 0)-K_{1}^{+}(\xi, y) \hat{\rho}_{n}^{+}+\int_{0}^{y+} K_{2}^{+}(\xi, y, z) \hat{v}_{n}^{+}(z) d z, \\
& \hat{w}_{n}^{-}(\xi, y)=e^{-i \beta-y} \hat{w}_{n}^{-}(\xi, 0)+K_{1}^{-}(\xi, y) \hat{\rho}_{n}^{-}-\int_{y_{-}}^{0} K_{2}^{-}(\xi, y, z) \hat{v}_{n}^{-}(z) d z,
\end{aligned}
$$

where

$$
\begin{aligned}
& K_{1}^{+}(\xi, y)=\frac{e^{\mathrm{i} \beta_{+} y_{+}}\left(e^{-\mathrm{i} \beta_{+} y}-e^{\mathrm{i} \beta_{+} y}\right)}{2 \mathrm{i} \beta_{+}}, \\
& K_{1}^{-}(\xi, y)=\frac{e^{-\mathrm{i} \beta-y-}\left(e^{\mathrm{i} \beta-y}-e^{-\mathrm{i} \beta-y}\right)}{2 \mathrm{i} \beta_{-}},
\end{aligned}
$$

and

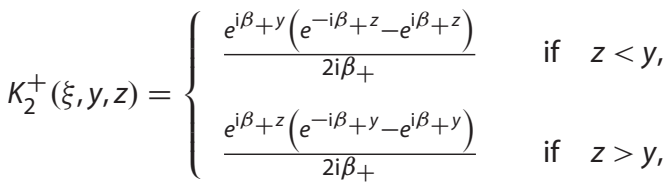

$$
\begin{aligned}
& K_{2}^{-}(\xi, y, z)= \begin{cases}\frac{e^{-\mathrm{i} \beta-z}\left(e^{-\mathrm{i} \beta-y}-e^{\mathrm{i} \beta-y}\right)}{2 \mathrm{i} \beta+} & \text { if } z<y, \\
\frac{e^{-\mathrm{i} \beta-y}\left(e^{-\mathrm{i} \beta-z}-e^{\mathrm{i} \beta-z}\right)}{2 \mathrm{i} \beta-} & \text { if } z>y,\end{cases}
\end{aligned}
$$

with

$$
\begin{aligned}
\hat{w}_{n}^{+}(\xi, 0)= & \hat{w}_{n}^{-}(\xi, 0)=\frac{-\mathrm{i}}{\beta_{+}+\beta_{-}} \times \\
& {\left[\hat{\varrho}_{n}+e^{-\mathrm{i} \beta_{-} y_{-}} \hat{\rho}_{n}^{-}-e^{\mathrm{i} \beta+y_{+}} \hat{\rho}_{n}^{+}+\int_{0}^{y_{+}} e^{\mathrm{i} \beta_{+} z} \hat{v}_{n}^{+}(z) \mathrm{d} z+\int_{y_{-}}^{0} e^{-\mathrm{i} \beta-z} \hat{v}_{n}^{-}(z) \mathrm{d} z\right] . }
\end{aligned}
$$

Remark 4.2

Under the assumption that $f$ has two continuous derivative, that is, $f \in C^{2}(\mathbb{R})$, we may follow the same techniques by Nicholls and Reitich [30] to show inductively, for $\varepsilon$ sufficiently small, that

$$
\left\|w_{n}^{+}\right\|_{H^{2}(\Omega)} \leq K_{+} \quad \text { and } \quad\left\|w_{n}^{-}\right\|_{H^{2}(\Omega)} \leq K_{-}, \quad \forall n \geq 0,
$$

where $K_{+}$and $K_{-}$are two positive constants independent of the index $n$. Therefore, the power series for $w^{+}$and $w^{-}$in (4.13) converge for sufficiently small $\varepsilon$.

\section{Concluding remarks}

We proposed in this paper a variational formulation for the scattering problem by an unbounded penetrable rough surface and derived the analytical solution when the scattering surface is assumed to be a sufficiently small and smooth perturbation of a plane surface.

In the first part, we reduced the scattering problem to a boundary value problem by using transparent boundary condition and proved the uniqueness and existence of the weak solution for the variational problem. In the second part, by using the transformed field expansion and Fourier transform, we converted the original two-dimensional problem with complex scattering surface into a sequence of one-dimensional two-point boundary value problems with decoupled boundary conditions in the frequency space for which the analytical solution can be explicitly expressed by using the integrated solution method.

Although the analytical solution given in Theorem 4.1 is of interest for many purposes, it is not very convenient to use in practice because of the lack of discrete Fourier transform on the infinite domain $\mathbb{R}$. In [24], we shall extend the numerical method in [32,33] to construct a practical algorithm for solving this problem.

An essential assumption we made is that $\operatorname{Im} \kappa_{ \pm}^{2} \neq 0$. A challenging problem is to study the infinite rough surface-scattering problem in lossless medium, that is, $\operatorname{Im} \kappa_{ \pm}^{2}=0$. Without energy decay, the capacity operator introduced in this paper is unbounded, and the proposed method can not be directly applied to this case. We hope to address these issues by studying the limiting absorption principle in the future. We also intend to extend the method to more complicated three-dimensional Maxwell's equations. 


\section{Appendix A. A two-point boundary value problem}

In this section, the integrated solution method is briefly introduced to solve a two-point boundary value problem. We refer to Zhang [47] for the details of the integrated solutions of ordinary differential equation system and two-point boundary value problems.

Consider the two-point boundary value problem

$$
\begin{gathered}
\mathbf{u}^{\prime}(y)+M(y) \mathbf{u}(y)=\mathbf{f}(y), \\
\left.A_{0} \mathbf{u}(y)\right|_{y=0}=\mathbf{r}_{0}, \\
\left.B_{1} \mathbf{u}(y)\right|_{y=L}=\mathbf{s}_{1},
\end{gathered}
$$

where $\mathbf{f}(y) \in \mathbb{C}^{m}$ are $m$-dimensional vector fields, $\mathbf{r}_{0} \in \mathbb{C}^{m_{1}}$ and $\mathbf{s}_{1} \in \mathbb{C}^{m_{2}}$ are given $m_{1}$-dimensional and $m_{2}$-dimensional vector fields, respectively, $M(y) \in \mathbb{C}^{m \times m}$ is an $m \times m$ matrix, and $A_{0} \in \mathbb{C}^{m_{1} \times m}$ and $B_{1} \in \mathbb{C}^{m_{2} \times m}$ are full rank matrices with $m_{1}+m_{2}=m$, that is, $\operatorname{rank} A_{0}=m_{1}$ and $\operatorname{rank} B_{1}=m_{2}$.

Let $\Phi(y)$ be the fundamental matrix of the system

$$
\begin{gathered}
\Phi^{\prime}(y)+M(y) \Phi(y)=\mathbf{0}, \\
\Phi(0)=l,
\end{gathered}
$$

where $l$ is the $m \times m$ identity matrix.

Theorem A. 1

Two-point boundary value problem (A.1)-(A.3) has a unique solution if and only if

$$
\operatorname{det}\left[\begin{array}{c}
A_{0} \\
B_{1} \Phi(L)
\end{array}\right] \neq 0 .
$$

Let the pair of functions $\{A(y), \mathbf{r}(y)\}$ and $\{B(y), \mathbf{s}(y)\}$ be the integrated solutions of problems (A.1)-(A.2) and (A.1)-(A.3), respectively, then there exist $D_{0}(A, y) \in \mathbb{C}^{m_{1} \times m_{1}}$ and $D_{1}(B, y) \in \mathbb{C}^{m_{2} \times m_{2}}$ such that

$$
\begin{gathered}
A^{\prime}=A M+D_{0} A, \quad A(0)=A_{0}, \\
\mathbf{r}^{\prime}=A \mathbf{f}+D_{0} \mathbf{r}, \quad \mathbf{r}(0)=\mathbf{r}_{0},
\end{gathered}
$$

and

$$
\begin{gathered}
B^{\prime}=B M+D_{1} B, \quad B(L)=B_{1}, \\
\mathbf{s}^{\prime}=B \mathbf{f}+D_{1} \mathbf{s}, \quad \mathbf{s}(L)=\mathbf{s}_{1} .
\end{gathered}
$$

Theorem A.2

If two-point boundary value problem (A.1)-(A.3) has a unique solution, then the matrix

$$
\left[\begin{array}{l}
A(y) \\
B(y)
\end{array}\right] \in \mathbb{C}^{m \times m}
$$

is nonsingular.

Theorem A.3

Two-point boundary value problem (A.1)-(A.3) is equivalent to the linear system

$$
\left[\begin{array}{l}
A(y) \\
B(y)
\end{array}\right] \mathbf{u}(y)=\left[\begin{array}{l}
\mathbf{r}(y) \\
\mathbf{s}(y)
\end{array}\right]
$$




\section{Appendix B. A transmission problem}

In this section, we discuss the solution of a transmission problem in details. Consider the second order boundary value problem

$$
\begin{aligned}
& u_{+}^{\prime \prime}+\lambda_{+}^{2} u_{+}=f_{+}, \quad 0<y<y_{+}, \\
& u_{-}^{\prime \prime}+\lambda_{-}^{2} u_{-}=f_{-}, \quad y_{-}<y<0,
\end{aligned}
$$

with the boundary conditions

$$
\begin{aligned}
& \partial_{y} u_{+}-\mathrm{i} \lambda_{+} u_{+}=g_{+} \quad \text { at } y=y_{+}, \\
& \partial_{y} u_{-}+\mathrm{i} \lambda_{-} u_{-}=g_{-} \quad \text { at } y=y_{-},
\end{aligned}
$$

and the jump conditions across the interface $y=0$ :

$$
\begin{gathered}
u_{+}(0)-u_{-}(0)=h_{1}, \\
\partial_{y} u_{+}(0)-\partial_{y} u_{-}(0)=h_{2} .
\end{gathered}
$$

We now convert the second-order differential equation into a first-order two-point boundary value problem. Let $v_{1}^{+}=u_{+}$and $v_{2}^{+}=u_{+}^{\prime}$, second-order boundary value problem (B.1) and (B.3) can be formulated into a first-order two-point boundary value problem:

$$
\begin{aligned}
& \mathbf{v}_{+}^{\prime}+M_{+} \mathbf{v}_{+}=\mathbf{f}_{+}, \\
& A_{0}^{+} \mathbf{v}_{+}(0)=u_{+}(0), \\
& B_{1}^{+} \mathbf{v}_{+}\left(y_{+}\right)=g_{+},
\end{aligned}
$$

where

$$
\mathbf{v}_{+}=\left[\begin{array}{l}
v_{1}^{+} \\
v_{2}^{+}
\end{array}\right], \quad \mathbf{f}_{+}=\left[\begin{array}{c}
0 \\
f_{+}
\end{array}\right], \quad M_{+}=\left[\begin{array}{cc}
0 & -1 \\
\lambda_{+}^{2} & 0
\end{array}\right],
$$

and

$$
A_{0}^{+}=[1,0], \quad B_{1}^{+}=\left[-\mathrm{i} \lambda_{+}, 1\right]
$$

Similarly, let Let $v_{1}^{-}=u_{-}$and $v_{2}^{-}=u_{-}^{\prime}$, second-order boundary value problem (B.2) and (B.4) can be formulated into a first-order two-point boundary value problem:

$$
\begin{aligned}
& \mathbf{v}_{-}^{\prime}+M_{-} \mathbf{v}_{-}=\mathbf{f}_{-}, \\
& A_{0}^{-} \mathbf{v}_{-}(0)=u_{-}(0), \\
& B_{1}^{-} \mathbf{v}_{-}\left(y_{-}\right)=g_{-},
\end{aligned}
$$

where

$$
\mathbf{v}_{-}=\left[\begin{array}{l}
v_{1}^{-} \\
v_{2}^{-}
\end{array}\right], \quad \mathbf{f}_{-}=\left[\begin{array}{c}
0 \\
f_{-}
\end{array}\right], \quad M_{-}=\left[\begin{array}{cc}
0 & -1 \\
\lambda_{-}^{2} & 0
\end{array}\right]
$$

and

$$
A_{0}^{-}=[1,0], \quad B_{1}^{-}=\left[\mathrm{i} \lambda_{-}, 1\right]
$$

Lemma B. 1

Given $f_{+} \in L^{1}\left(0, y_{+}\right), u_{+}(0)$, and $g_{+} \in \mathbb{R}$. Two-point boundary value problem (B.7)-(B.9) has a unique solution given by

$$
u_{+}(y)=e^{\mathrm{i} \lambda+y} u_{+}(0)-K_{1}^{+}(y) g_{+}+\int_{0}^{y+} K_{2}^{+}(y, z) f_{+}(z) d z
$$


where

$$
K_{1}^{+}(y)=\frac{e^{\mathrm{i} \lambda+y+}\left(e^{-\mathrm{i} \lambda+y}-e^{\mathrm{i} \lambda+y}\right)}{2 \mathrm{i} \lambda_{+}}
$$

and

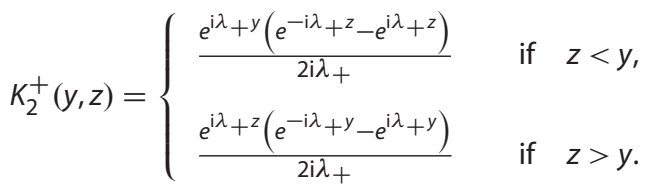

Proof

Because $M_{+}$is a nonsingular matrix, there exists a nonsingular matrix $Q_{+}$such that

$$
Q_{+}^{-1} M_{+} Q_{+}=N_{+},
$$

where

$$
N_{+}=\left[\begin{array}{cc}
-\mathrm{i} \lambda_{+} & 0 \\
0 & \mathrm{i} \lambda_{+}
\end{array}\right], \quad Q_{+}=\left[\begin{array}{cc}
1 & 1 \\
\mathrm{i} \lambda_{+} & -\mathrm{i} \lambda_{+}
\end{array}\right], \quad \text { and } \quad Q_{+}^{-1}=\frac{1}{2 \mathrm{i} \lambda_{+}}\left[\begin{array}{cc}
\mathrm{i} \lambda_{+} & 1 \\
\mathrm{i} \lambda_{+} & -1
\end{array}\right]
$$

A simple calculation yields

$$
\operatorname{det}\left[\begin{array}{c}
A_{0}^{+} \\
B_{1}^{+} e^{M_{+}}
\end{array}\right]=\left|\begin{array}{cc}
1 & 0 \\
\mathrm{i} \lambda_{+} e^{\mathrm{i} \lambda_{+}} & -e^{-\mathrm{i} \lambda_{+}}
\end{array}\right|=-e^{-\mathrm{i} \lambda_{+}} \neq 0 .
$$

It follows from Theorem A.1 that two-point boundary value problem (B.7)-(B.9) has a unique solution.

Let $\left\{A_{+}(y), r_{+}(y)\right\}$ and $\left\{B_{+}(y), s_{+}(y)\right\}$ be the integrated solutions of problems (B.7), (B.8) and (B.7), (B.9), respectively. Taking

$$
D_{0}^{+}=\mathrm{i} \lambda_{+}, \quad D_{1}^{+}=-\mathrm{i} \lambda_{+},
$$

we obtain from (A.7)-(A.10) that the integrated solutions satisfy

$$
\begin{aligned}
& A_{+}^{\prime}=A_{+} M_{+}+\mathrm{i} \lambda_{+} A_{+}, \quad A_{+}(0)=A_{0}^{+}, \\
& r_{+}^{\prime}=A_{+} \mathbf{f}_{+}+\mathrm{i} \lambda_{+} r_{+}, \quad r_{+}(0)=u_{+}(0),
\end{aligned}
$$

and

$$
\begin{gathered}
B_{+}^{\prime}=B_{+} M_{+}-\mathrm{i} \lambda_{+} B_{+}, \quad B_{+}\left(y_{+}\right)=B_{1}^{+}, \\
s_{+}^{\prime}=B_{+} \mathbf{f}_{+}-\mathrm{i} \lambda_{+} s_{+}, \quad s_{+}\left(y_{+}\right)=g_{+} .
\end{gathered}
$$

Upon solving the aforementioned initial value problem, we obtain the integrated solutions

$$
\begin{aligned}
& A_{+}=\left[A_{1}^{+}, A_{2}^{+}\right]=\frac{1}{2 \mathrm{i} \lambda_{+}}\left[\mathrm{i} \lambda_{+}\left(1+e^{2 \mathrm{i} \lambda+y}\right), 1-e^{2 \mathrm{i} \lambda_{+} y}\right], \\
& B_{+}=\left[B_{1}^{+}, B_{2}^{+}\right]=\left[-\mathrm{i} \lambda_{+}, 1\right], \\
& r_{+}=e^{\mathrm{i} \lambda}+y_{+}(0)+\int_{0}^{y} e^{\mathrm{i} \lambda} \lambda_{+}^{(y-z)} A_{2}^{+}(z) f_{+}(z) \mathrm{d} z \\
& s_{+}=e^{\mathrm{i} \lambda_{+}\left(y_{+}-y\right)} g_{+}-\int_{y}^{y_{+}} e^{\mathrm{i} \lambda_{+}(z-y)} f_{+}(z) \mathrm{d} z .
\end{aligned}
$$

It follows from Theorem A.3 that two-point boundary value problem (B.7)-(B.9) is equivalent to the linear system

$$
\left[\begin{array}{ll}
A_{1}^{+} & A_{2}^{+} \\
B_{1}^{+} & B_{2}^{+}
\end{array}\right]\left[\begin{array}{l}
u_{+} \\
u_{+}^{\prime}
\end{array}\right]=\left[\begin{array}{c}
r_{+} \\
s_{+}
\end{array}\right] .
$$


An application of Gram's rule yields

$$
u_{+}=\frac{r_{+} B_{2}^{+}-s_{+} A_{2}^{+}}{A_{1}^{+} B_{2}^{+}-B_{1}^{+} A_{2}^{+}} \text {. }
$$

A simple calculation yields

$$
A_{1}^{+} B_{2}^{+}-B_{1}^{+} A_{2}^{+}=1 \text {. }
$$

Substituting (B.18)-(B.21) into (B.22), we deduce (B.13).

Lemma B.2

Given $f_{-} \in L^{1}\left(y_{-}, 0\right), u_{-}(0)$, and $g_{-} \in \mathbb{R}$. Two-point boundary value problem (B.10)-(B.12) has a unique solution given by

$$
u_{-}(y)=e^{-\mathrm{i} \lambda-y_{-}} u_{-}(0)+K_{1}^{-}(y) g_{-}-\int_{y_{-}}^{0} K_{2}^{-}(y, z) f_{-}(z) \mathrm{d} z,
$$

where

$$
K_{1}^{-}(y)=\frac{e^{-\mathrm{i} \lambda-y-}\left(e^{\mathrm{i} \lambda-y}-e^{-\mathrm{i} \lambda-y}\right)}{2 \mathrm{i} \lambda_{-}}
$$

and

$$
K_{2}^{-}(y, z)= \begin{cases}\frac{e^{-\mathrm{i} \lambda-z}\left(e^{-\mathrm{i} \lambda-y}-e^{\mathrm{i} \lambda-y}\right)}{2 \mathrm{i} \lambda+} & \text { if } z<y, \\ \frac{e^{-\mathrm{i} \lambda-y}\left(e^{-\mathrm{i} \lambda-z}-e^{\mathrm{i} \lambda-z}\right)}{2 \mathrm{i} \lambda-} & \text { if } z>y .\end{cases}
$$

Proof

The proof is similar to the one for Lemma B.1. It can be verified that

$$
\operatorname{det}\left[\begin{array}{c}
A_{0}^{-} \\
B_{1}^{-} e^{M_{-}}
\end{array}\right]=\left|\begin{array}{cc}
1 & 0 \\
\mathrm{i} \lambda_{-} e^{-\mathrm{i} \lambda_{-}} & e^{-\mathrm{i} \lambda_{-}}
\end{array}\right|=e^{-\mathrm{i} \lambda_{-}} \neq 0,
$$

so Theorem A.1 implies that problem (B.10)-(B.12) has a unique solution.

Let $\left\{A_{-}(y), r_{-}(y)\right\}$ and $\left\{B_{-}(y), s_{-}(y)\right\}$ be the integrated solutions of problems (B.10), (B.11) and (B.10), (B.12), respectively. Taking

$$
D_{0}^{-}=-\mathrm{i} \lambda_{-}, \quad D_{1}^{-}=\mathrm{i} \lambda_{-},
$$

we obtain from (A.7)-(A.10) that the integrated solutions satisfy

$$
\begin{aligned}
& A_{-}^{\prime}=A_{-} M_{-}-\mathrm{i} \lambda_{-} A_{-}, \quad A_{-}(0)=A_{0}^{-}, \\
& r_{-}^{\prime}=A_{-} \mathbf{f}_{-}-\mathrm{i} \lambda_{-} r_{-}, \quad r_{-}(0)=u_{-}(0),
\end{aligned}
$$

and

$$
\begin{gathered}
B_{-}^{\prime}=B_{-} M_{-}+\mathrm{i} \lambda_{-} B_{-}, \quad B_{-}\left(y_{-}\right)=B_{1}^{-}, \\
s_{-}^{\prime}=B_{-} \mathbf{f}_{-}+\mathrm{i} \lambda_{-} s_{-}, \quad s_{-}\left(y_{-}\right)=g_{-} .
\end{gathered}
$$

Upon solving the aforementioned initial value problem, we obtain the integrated solutions

$$
\begin{gathered}
A_{-}=\left[A_{1}^{-}, A_{2}^{-}\right]=\frac{1}{2 \mathrm{i} \lambda_{-}}\left[\mathrm{i} \lambda_{-}\left(e^{-2 \mathrm{i} \lambda_{-} y}+1\right), e^{-2 \mathrm{i} \lambda_{-} y}-1\right], \\
B_{-}=\left[B_{1}^{-}, B_{2}^{-}\right]=\left[\mathrm{i} \lambda_{-}, 1\right] \\
r_{-}=e^{-\mathrm{i} \lambda_{-} y_{u_{-}}(0)+\int_{0}^{y} e^{-\mathrm{i} \lambda_{-}(y-z)} A_{2}^{-}(z) f_{-}(z) \mathrm{d} z}
\end{gathered}
$$




$$
s_{-}=e^{-\mathrm{i} \lambda_{-}\left(y_{-}-y\right)} g_{-}-\int_{y}^{y_{-}} e^{-\mathrm{i} \lambda_{+}(z-y)} f_{-}(z) \mathrm{d} z
$$

An application of Gram's rule yields

$$
u_{-}=\frac{r_{-} B_{2}^{-}-s_{-} A_{2}^{-}}{A_{1}^{-} B_{2}^{-}-B_{1}^{-} A_{2}^{-}}
$$

A simple calculation yields

$$
A_{1}^{-} B_{2}^{-}-B_{1}^{-} A_{2}^{-}=1
$$

Substituting (B.28)-(B.31) into (B.32), we deduce (B.23).

To completely determine the solutions $u_{+}(y)$ and $u_{-}(y)$, it is required to compute $u_{+}(0)$ and $u_{-}(0)$, which can be obtained by using jump conditions (B.5) and (B.6). Simple calculations yield

$$
\begin{aligned}
& \partial_{y} K_{1}^{+}(0)=-e^{\mathrm{i} \lambda+y_{+}}, \quad \partial_{y} K_{2}^{+}(0, z)=-e^{\mathrm{i} \lambda+z_{+},}, \\
& \partial_{y} K_{1}^{-}(0)=e^{-\mathrm{i} \lambda-y_{-}}, \quad \partial_{y} K_{2}^{-}(0, z)=-e^{-\mathrm{i} \lambda_{-} z},
\end{aligned}
$$

which give

$$
\begin{aligned}
& \partial_{y} u_{+}(0)=\mathrm{i} \lambda_{+} u_{+}(0)+e^{\mathrm{i} \lambda+y_{+}} g_{+}-\int_{0}^{y_{+}} e^{\mathrm{i} \lambda_{+} z} f_{+}(z) \mathrm{d} z \\
& \partial_{y} u_{-}(0)=-\mathrm{i} \lambda_{-} u_{-}(0)+e^{-\mathrm{i} \lambda_{-} y_{-}} g_{-}+\int_{y_{-}}^{0} e^{-\mathrm{i} \lambda_{-} z} f_{-}(z) \mathrm{d} z
\end{aligned}
$$

It follows that from jump conditions (B.5) and (B.6) we obtain

$$
\begin{aligned}
u_{-}(0)= & \frac{-\mathrm{i}}{\lambda_{+}+\lambda_{-}} \times \\
& {\left[h_{2}-\mathrm{i} \lambda_{+} h_{1}+e^{-\mathrm{i} \lambda_{-} y_{-}} g_{-}-e^{\mathrm{i} \lambda_{+} y_{+}} g_{+}+\int_{0}^{y_{+}} e^{\mathrm{i} \lambda_{+}{ }^{z}} f_{+}(z) \mathrm{d} z+\int_{y_{-}}^{0} e^{-\mathrm{i} \lambda_{-} z} f_{-}(z) \mathrm{d} z\right] }
\end{aligned}
$$

and

$$
u_{+}(0)=u_{-}(0)+h_{1}
$$

Theorem B.1

Given $f_{-} \in L^{1}\left(y_{-}, 0\right), f_{+} \in L^{1}\left(0, y_{+}\right)$, and $g_{ \pm} \in \mathbb{R}$. Transmission problem (B.1)-(B.6) has a unique solution explicitly expressed by (B.13) and (B.23) with $u_{ \pm}(0)$ given by (B.33)-(B.34).

\section{Acknowledgements}

This research was supported in part by the National Science Foundation grants DMS-0914595, DMS-1042958, and DMS-0915066 and by the Air Force Office of Scientific Research grant FA9550-08-1-0416.

\section{References}

1. Ogilvy JA. Theory of wave Scattering from Random Rough Surfaces. Adam Hilger: Bristol, 1991.

2. Voronovich AG. Wave Scattering from Rough Surfaces. Springer: Berlin, 1994.

3. Saillard M, Sentenac A. Rigorous solutions for electromagnetic scattering from rough surfaces. Waves Random Media 2001; 11:R103-R137.

4. Warnick K, Chew WC. Numerical simulation methods for rough surface scattering. Waves Random Media 2001; 11:R1-R30.

5. DeSanto JA. Scattering by rough surfaces. In Scattering: Scattering and Inverse Scattering in Pure and Applied Science, Pike R, Sabatier P (eds). Academic Press: New York, 2002; 15-36.

6. Elfouhaily TM, Guerin CA. A critical survey of approximate scattering wave theories from random rough surfaces. Waves Random Media 2004; 14:R1-R40.

7. Chandler-Wilde SN, Zhang B. A uniqueness result for scattering by infinte rough surfaces. SIAM Journal on Applied Mathematics 1998; 58:1774-1790.

8. Arens T, Hohage T. On radiation conditions for rough surface scattering problems. SIAM Journal on Applied Mathematics 2005; 70:839-847.

9. Ritterbusch S. Coercivity and the calderon operator on an unbounded domain. PhD Thesis, Karlsruhe Institute of Technology, 2009.

10. Chandler-Wilde SN, Elschner J. Variational approach in weighted Sobolev spaces to scattering by unbounded rough surfaces. SIAM Journal on Mathematical Analysis 2010; 42:2554-2580.

11. Chandler-Wilde SN, Monk P. Existence, uniqueness and variational methods for scattering by unbounded rough surfaces. SIAM Journal on Mathematical Analysis 2005; 37:598-618. 
12. Chandler-Wilde SN, Monk P, Thomas M. The mathematics of scattering by unbounded, rough, inhomogeneous layers. Journal of Computational and Applied Mathematics 2007; 204:549-559.

13. Lechleiter A, Ritterbusch S. A variational method for wave scattering from penetrable rough layer. IMA Journal on Applied Mathematics 2010; 75:366-391.

14. Li P, Wu H, Zheng W. Electromagnetic scattering by unbounded rough surfaces. Mathematical Methods in the Applied Sciences; to appear.

15. Chandler-Wilde SN, Heinemeyer E, Potthast R. A well-posed integral equation formulating for three-dimensional rough surface scattering. Proceedings of the Royal Society A 2006; 462:3683-3705.

16. Chandler-Wilde SN, Ross CR, Zhang B. Scattering by infinite one-dimensional rough surfaces. Proceedings of the Royal Society of London Series A 1999; 455:3767-3787.

17. Zhang B, Chandler-Wilde SN. Acoustic scattering by an inhomogeneous layer on a rigid plate. SIAM Journal on Applied Mathematics 1998; 58:1931-1950.

18. Zhang B, Chandler-Wilde SN. Integral equation methods for scattering by infinite rough surfaces. Mathematical Methods in the Applied Sciences 2003; 26:463-488.

19. DeSanto JA, Martin PA. On angular-spectrum representations for scattering by infinite rough surfaces. Wave Motion 1996; 24:421-433.

20. DeSanto JA, Martin PA. On the derivation of boundary integral equations for scattering by an infinite one-dimensional rough surface. Journal of Acoustic Society of America A 1997; 102:67-77.

21. DeSanto JA, Martin PA. On the derivation of boundary integral equations for scattering by an infinite two-dimensional rough surface. Journal of Mathematical Physics 1998; 39:894-912.

22. Chandler-Wilde SN, Zhang B. Electromagnetic scattering by an inhomogeneous conducting or dielectric layer on a perfectly conducting plate. Proceedings of the Royal Society of London Series A 1998; 454:519-542.

23. Li P. Coupling of finite element and boundary integral method for electromagnetic scattering in a two-layered medium. Journal of Computational Physics 2010; 229:481-497.

24. He Y, Li P, Shen J. An efficient and robust Fourier-Hermite method for the scattering by an unbounded rough surface. in preparation.

25. Bruno O, Reitich F. Numerical solution of diffration problems: a method of variation of boundaries. Journal of Optical Society of America A 1993; 10:1168-1175.

26. Bruno O, Reitich F. Numerical solution of diffration problems: a method of variation of boundaries. II. Finitely conducting gratings, Padé approximants, and singularities. Journal of Optical Society of America A 1993; 10:2307-2316.

27. Bruno O, Reitich F. Numerical solution of diffration problems: a method of variation of boundaries. III. Doubly periodic gratings. Journal of Optical Society of America A 1993; 10:2551-2562.

28. Bruno O, Reitich F. Boundary-variation solutions for bounded-obstacle scattering problems in three dimensions. Journal of Acoustical Society of America 1998; 104:2579-2583.

29. Bruno O, Reitich F. High-order boundary perturbation methods. In Mathematical Modeling in Optical Sciences, Vol. 22, Bao G, Cowsar L, Masters W (eds), Frontiers in Applied Mathematics Series. SIAM: Philadelphia, 2001; 71-109.

30. Nicholls DP, Reitich F. Shape deformations in rough surface scattering: cancellations, conditioning, and convergence. Journal of Optical Society of America A 2004; 21:590-605.

31. Nicholls DP, Reitich F. Shape deformations in rough surface scattering: improved algorithms. Journal of Optical Society of America A $2004 ; 21: 606-621$.

32. Nicholls DP, Shen J. A stable high-order method for two-dimensional bounded-obstacle scattering. SIAM Journal on Scientific Computing 2006; 28:1398-1419.

33. Fang Q, Nicholls DP, Shen J. A stable, high-order method for three-dimensional bounded-obstacle, acoustic scattering. Journal of Computational Physics 2007; 224:1145-1169.

34. Bao G. Finite element approximation of time harmonic waves in periodic structures. SIAM Journal on Numerical Analysis 1995; 32:1155-1169.

35. Bao G. Variational approximation of Maxwell's equations in biperiodic structures. SIAM Journal on Applied Mathematics 1997; 57:364-381.

36. Ammari H, Bao G, Wood A. An integral equation method for the electromagnetic scattering from cavities. Mathematical Methods in the Applied Sciences 2000; 23:1057-1072.

37. Ammari H, Bao G, Wood A. Analysis of the electromagnetic scattering from a cavity. Japan Journal on Industrial and Applied Mathematics 2001; 19:301-308.

38. Van T, Wood A. Finite element analysis for 2-D cavity problem. IEEE Transactions on Antennas and Propagation 2003; 51:1-8.

39. Wood A. Analysis of electromagnetic scattering from an overfilled cavity in the ground plane. Journal of Computational Physics 2006; 215:630-641.

40. Ammari H, Bao G. Mathematical modeling of near-field optics. Journal of Mathematical Analysis and Applications 2002; 265:430-446.

41. Ammari H, Bao G. Maxwell's equations in a perturbed periodic structure. Advances in Computational Mathematics 2002; 16:99-112.

42. Colton D, Kress R. Integral Equation Methods in Scattering Theory. Wiley: New York, NY, 1983.

43. Colton D, Kress R. Inverse Acoustic and Electromagnetic Scattering Theory, 2nd ed, Appl. Math. Sci. 93. Springer-Verlag: Berlin, 1998.

44. Nédélec J-C. Acoustic and Electromagnetic Equations: Integral Representations for Harmonic Problems. Springer: New York, 2000.

45. Monk P. Finite Element Methods for Maxwell's Equations. Oxford University Press: Oxford, U. K., 2003.

46. Gilbarg D, Trudinger N. Elliptic Partial Differential Equations of Second Order. Springer: New York, 1983.

47. Zhang G-Q. Integrated solutions of ordinary differential equation system and two-point boundary value problems, I. Integrated solution method. Journal of Computational Mathematics 1981; 3:245-254. 